\title{
AFINAL, ONDE CHEGAMOS? AVALIAÇÃO DA CONSTRUÇÃO DE UM MODELO DE GOVERNANÇA COLABORATIVO NA PERSPECTIVA DE SEUS PARTICIPANTES
}

\author{
Carolina Lopes Araújo', Janaína Siqueira Silva², Lia Teles Vasconcelos ${ }^{3}$ e Eliane Almeida do \\ Carmo 4 \\ ${ }^{1 y 2}$ Universidade de Brasília, Brasil. ${ }^{1}$ carolinaaraujo@unb.br; ${ }^{2}$ siqueira.janaina6@gmail.com \\ ${ }^{3}$ MARE - Universidade Nova de Lisboa, Portugal. Itv@fct.unl.pt \\ ${ }^{4}$ Fundação Osvaldo Cruz (FIOCRUZ - Brasília), Brasil. eliane.adm@gmail.com
}

\begin{abstract}
Resumo. O artigo focaliza a avaliação dos stakeholders quanto ao Projeto MARGov - Governança Colaborativa de Áreas Marinhas Protegidas, desenvolvido na região de Sesimbra, Portugal. A participação social é requisito para a sustentabilidade, mas o engajamento dos stakeholders é condicionado pela percepção do valor e dos resultados dos esforços coletivos. A fim de conhecer aspectos importantes para o engajamento dos participantes e, logo, do sucesso do Projeto MARGov, este estudo aplicou a análise de conteúdo, a partir de categorias temáticas e de categorias avaliativas inspiradas na Análise SWOT, sobre entrevistas semiestruturadas nas quais 17 stakeholders de seis diferentes grupos sociais avaliaram o Projeto MARGov, considerando aspectos que influenciaram seu processo (execução) e seus resultados. As análises revelaram que, apesar de as avaliações negativas prevalecerem nos textos, essas se pautaram, majoritariamente, sobre elementos do contexto que revelam a medida do desafio de se conciliar interesses numa área ambiental altamente antropizada. Quanto aos aspectos internos do projeto, avaliações positivas predominam nas entrevistas e representam o reconhecimento da importância do Projeto MARGov pelos participantes. Não obstante, aspectos internos avaliados negativamente merecem atenção pois sinalizam fragilidades e apontam para oportunidades de aprimoramento do Projeto.
\end{abstract}

Palavras-chave: Participação Social; Sustentabilidade; Avaliação por Stakeholders; Margov; SWOT.

\section{SO, WHERE DID WE GET? EVALUATION OF A COLLABORATIVE PROJECT FROM THE PERSPECTIVE OF ITS PARTICIPANTS}

\begin{abstract}
Social participation is a prerequisite for sustainability, but stakeholder engagement is conditioned by their perception of value and results of collective efforts. The MARGov Project Collaborative Governance of Marine Protected Areas was developed in the region of Sesimbra Portugal. This paper focus participants' assessment of the MARGov Project, aiming to reveal aspects favoring stakeholder engagement and, therefore, MARGov achievements. Thus, we've applied content analysis, based on thematic categories and on evaluative categories inspired by the SWOT Analysis, on semi-structured interviews in which 17 stakeholders from six different social groups evaluated the MARGov Project, considering aspects that influenced its process (execution) and its results. The analyzes revealed that, although the negative evaluations prevail in the texts, these were mostly based on elements of the context that reveal the measure of the challenge of reconciling interests in a highly anthropized environmental area. As for the internal aspects of the project, positive evaluations predominate in the interviews and represent the participants' recognition about the importance of the MARGov Project. Nevertheless, negatively evaluated internal aspects deserve attention because they sign weaknesses and point out opportunities for improving the Project.
\end{abstract}

Keywords: Social Participation; Sustainability; Stakeholder Assessment; Margov; SWOT. 


\section{INTRODUÇÃO}

As Áreas Marinhas Protegidas (AMP) são mecanismos de suma importância para a conservação da biodiversidade, para assegurar a pesca sustentável e a preservação da cultura litorânea local. O Parque Marinho Professor Luiz Saldanha (PMPLS) é uma área de preservação ambiental permanente, criado em 1998, que integra o Parque Natural da Arrábida (PNA), situado na península de Setúbal, Área Metropolitana de Lisboa, Portugal. Em 2005, quando o Plano de Ordenamento do Parque Natural da Arrábida (POPNA) entrou em vigor, vieram a público inúmeras e intensas manifestações de insatisfação provenientes de diferentes grupos sociais ligados ao Parque. A necessidade de gerir os conflitos e viabilizar um pacto social pelo uso sustentável dos recursos naturais no PMPLS oportunizou o desenvolvimento do Projeto MARGov, foco deste trabalho.

O Projeto MARGov mobilizou stakeholders e estimulou a articulação de conhecimentos e saberes com o intuito de conciliar perspectivas e contribuir para o desenvolvimento de um Modelo de Governança Colaborativa (MGC) pelo qual cada parceiro pudesse atuar como um agente de gestão sustentável do Parque (Stratoudakis, Farrall, \& Vasconcelos, 2018).

Neste artigo, a análise de conteúdo de entrevistas coletadas junto aos principais participantes do Projeto MARGov permitiu conhecer as avaliações desses atores quanto a aspectos internos e externos do Projeto. Essas avaliações revelaram a percepção dos participantes quanto aos processos e resultados do Projeto MARGov e desvelaram aspectos importantes que ajudaram a condicionar o engajamento social de crucial importância na construção coletiva desse modelo de gestão colaborativa até então inédito em Portugal.

\section{PARTICIPAÇÃO SOCIAL E DEMOCRACIA: A EVOLUÇÃO DE UM CONCEITO}

Vê-se uma evolução da ideia e da prática da democracia que migram da representatividade para uma abordagem de participação ativa de atores sociais nas questões que the sejam concernentes. Já na Conferência de Estocolmo, em 1972, se pautou o compromisso de promover a ativa participação cidadã como meio para se associar a sociedade civil com a gestão e o controle ambiental (vide Recomendação 97, alínea (a)) (United Nations General Assembly, 1972). Mas foi a partir da Conferência das Nações Unidas para o Desenvolvimento e Meio Ambiente - a ECO-92, que a participação social se apresenta como um imperativo para as questões ambientais. A Declaração do Rio (United Nations, 
1992) proclama, em seu Princípio 10, que "o melhor modo de tratar as questões ambientais é com a participação de todos os cidadãos interessados"1. A Agenda 21, no Capítulo 8, assegura a efetiva participação social nos assuntos relativos a desenvolvimento e meio ambiente (United Nations, 1992). O "Futuro que Queremos", documento resultado da Rio+20, em seu parágrafo 13 , reconhece "como fundamental para o desenvolvimento sustentável as oportunidades dos povos em serem atores de suas vidas e de seu futuro, de participarem das tomadas de decisões e de expressarem suas preocupações" (United Nations General Assembly, 2012)2. Na Europa, a essas diretrizes da Organização das Nações Unidas, se agrega a Convenção de Aarhus sobre Acesso à Informação, Participação do Público no Processo de Tomada de Decisão e Acesso à Justiça em Matéria de Ambiente que, assinada pelos países da União Europeia em 1998, entra em vigor em 2001 assegurando a todos o direito de participar das decisões relativas ao ambiente.

Carneiro (2011, p. 325) explica que há justificativas morais e legais, mas também pragmáticas, para que seja imperativa a participação social nas questões relativas ao meio ambiente. O envolvimento da população tem se mostrado imprescindível para qualquer iniciativa de preservação ambiental.

Áreas de preservação ambiental permanente precisam conciliar as imediatas demandas por desenvolvimento da população local com os amplos interesses de preservação ambiental. O desafio se avoluma na medida em que essas áreas se instalam em paisagens com significativa presença humana. As pressões antrópicas sobre os recursos naturais, as disputas por acesso aos recursos naturais de uso comum e a diversidade dos stakeholders a serem envolvidos nas decisões sobre a área incrementam a complexidade dos processos de participação social (Stratoudakis, Farrall, \& Vasconcelos, 2018). Mas os riscos de não se alcançar um pacto entre os atores sociais para a preservação das zonas de alto interesse e valor ambiental podem se traduzir em práticas recorrentes de irregularidades ou infrações na área ou, até mesmo, no esvaziamento dos marcos regulatórios do uso das áreas de preservação.

\footnotetext{
${ }^{1}$ Tradução livre de: "Environmental issues are best handled with the participation of all concerned citizens, at the relevant level".

${ }^{2}$ Tradução utilizada pelo Ministério do Meio Ambiente brasileiro, do original: "We recognize that opportunities for people to influence their lives and future, participate in decision-making and voice their concerns are fundamental for sustainable development".
} 
Portanto, a promoção da participação ativa dos atores locais num arranjo colaborativo de corresponsabilidade pelo monitoramento e gestão da área de elevada riqueza ambiental, bem como a atuação para a redução dos conflitos que surgem quando da implementação de uma área de preservação permanente, exigem ferramentas adequadas e inovadoras de intervenção em contextos complexos de multi-atores (Stratoudakis, Farrall, \& Vasconcelos, 2018).

\section{O PARQUE MARINHO PROFESSOR LUIZ SALDANHA E O PARQUE NATURAL DA ARRÁBIDA}

Foi nesse contexto de crescentes exigências por participação social que foi implementado o Parque Marinho Professor Luiz Saldanha (PMPLS), criado em 1998 por força de Decreto Regulamentar no.23/98, acrescentando a zona costeira do litoral marinho aos limites do Parque Natural da Arrábida (PNA), criado em 1976, e "complementando no meio marinho os objetivos de conservação da natureza subjacentes à criação do Parque" (Ministério do Ambiente, 1998).

O Parque Marinho Professor Luiz Saldanha, localiza-se na região da Vila de Sesimbra, no distrito de Setúbal, na Área Metropolitana de Lisboa. O Parque é constituído por uma área com cerca de $53 \mathrm{~km}^{2}$ que resguarda uma elevada biodiversidade e contém zonas de reprodução de várias espécies de peixes, tanto de interesse comercial - como sardinha, linguado, carapau e outras - e, também, de interesse de preservação, como o cavalo marinho (Gonçalves, et al., 2015, p. 7). A pesca é a principal atividade de interesse econômico ali realizada, mas atividades marítimo-turísticas também são relevantes. Dessas atividades, Stratoudakis et al. (2015, p. 168) destacam, por significativa ocorrência, o mergulho autônomo (Scuba Diving), pesca lúdica apeada e embarcada e a náutica recreativa.

Carneiro (2011, p. 326) explica que nos sete primeiros anos de existência, o PMPLS não dispunha de regulação específica ou de um plano de gestão que promovessem mudanças na utilização do ambiente marinho. Foi com a entrada em vigor do Plano de Ordenamento do Parque Natural da Arrábida (POPNA), em 2005, que as restrições de uso se impuseram na zona do Parque Marinho. O desenvolvimento do POPNA contou com consultas públicas ao longo dos anos de 2003 e 2004, em conformidade com as diretrizes da legislação ambiental de Portugal. Mas as restrições e condicionalidades impostas às atividades tradicionalmente realizadas na área do Parque, junto com as vicissitudes ocorridas nos 
processos de consulta pública, resultaram em insatisfação e expressa oposição pública ao POPNA por diversos stakeholders. Tal situação de tensão evoluiu para a perda de confiança nas relações entre usuários e a gestão do Parque, resultando em conflitos que se fizeram sentir amplamente (Carneiro, 2011; Stratoudakis, Fernández, Henriques, Martins, \& Martins, 2015; Stratoudakis, Farrall, \& Vasconcelos, 2018).

\section{O PROJETO MARGOV: GOVERNANÇA COLABORATIVA DE ÁREAS MARINHAS PROTEGIDAS}

O Projeto MARGov de Governança Colaborativa de Áreas Marinhas Protegidas buscou superar os conflitos e desconfianças geradas com a implementação do PMPLS e sua regulação pelo POPNA e colaborar na construção de discursos consensuados. O MARGov objetivou contribuir para a implementação de um Modelo de Governança Colaborativa (MGC) pelo qual cada parceiro pudesse atuar como um agente de gestão sustentável do Parque (Stratoudakis, Farrall, \& Vasconcelos, 2018). Desde o início, a proposta do MARGov buscou mobilizar os stakeholders, especialmente os grupos insatisfeitos com a implementação do POPNA, com vistas a conciliar perspectivas articulando conhecimentos tradicionais e técnico-científicos sobre a área e promovendo a troca de informações, de boas práticas e de experiências. Desse modo, buscou-se favorecer o aprendizado mútuo, a compreensão da complexidade das questões relativas à preservação da área e a participação ativa dos atores implicados com a sustentabilidade do PMPLS (Vasconcelos, Coelho, Pereira, Sá, \& Costa, 2011). A possibilidade de replicação da experiência para outras áreas marinhas protegidas também foi um requisito do MARGov.

Stratoudakis et al. (2018) distinguem três fases de intervenção do Projeto MARGov. A primeira fase, em que se desenvolveu o Projeto MARGov, de 2008 a 2011, marcada pelo engajamento e a mobilização dos stakeholders; a segunda fase, de 2012 a 2014, denominada Pós-MARGov na sequência da primeira e implementada por vontade expressa do núcleo do Projeto, mas de atuação informal, porém estruturada e envolvendo múltiplos atores; e a terceira fase, de 2014 a 2018 marcada pela institucionalização e legitimação do grupo de stakeholders que passa a compor o Grupo do Mar - um grupo de consulta e de trabalho integrado ao Conselho Estratégico do Parque Natural da Arrábida.

Em 2011, o Projeto MARGov, no formato proposto inicialmente, foi concluído. No entanto, a equipe MARGov (grupo nuclear de facilitadores e investigadores) que acompanhou todo o projeto ao longo dos Fóruns Abertos solicitou que se continuasse o processo para a 
consolidação do trabalho que tinha sido desenvolvido e dos avanços nos consensos. Deste modo, foi criado o Pós-MARGov, e competiu à equipe MARGov facilitar reuniões e coordenar os trabalhos. Em 2014, o grupo foi convidado pelo Instituto da Conservação da Natureza (ICN) para integrar o grupo de aconselhamento para o Mar do Conselho Estratégico desse Instituto, tendo, portanto, se institucionalizado. Constata-se que parte das propostas derivadas dos consensos construídos ao longo da intervenção ganharam autonomia e sustentabilidade e acabaram integradas às diretrizes e práticas de gestão do Parque.

O foco deste trabalho reside na avaliação dos participantes quanto ao processo (à execução) e os resultados da primeira fase do Projeto MARGov. Sabendo-se que a manutenção do engajamento dos participantes é condicionada pelo reconhecimento da criação de valor do esforço coletivo e pelo alcance de resultados compartilhados (Thomson \& James, 2006), buscou-se analisar a avaliação dos participantes quanto aspectos internos e externos do Projeto MARGov. A análise das avaliações dos stakeholders se justifica, mesmo após a conclusão do Projeto, por ser importante conhecer as percepções (avaliações) que ajudaram a condicionar o engajamento dos stakeholders, tendo sido a manutenção desse engajamento crucial para que o Projeto pudesse se desdobrar em sua segunda e terceira fases.

\section{METODOLOGIA}

O corpus de pesquisa deste trabalho é composto pelo texto integral transcrito de entrevistas semiestruturadas coletadas junto a 17 participantes envolvidos na primeira fase do Projeto MARGov. As etapas do desenvolvimento da análise de conteúdo, conforme propostas por Bardin (2016, p. 132) orientaram o tratamento e a análise dos dados.

\subsection{Coleta de Dados}

As entrevistas foram coletadas por membros da equipe do Projeto MARGov, no período de julho a setembro de 2011, isto é, na transição da primeira para a segunda fase do Projeto. As entrevistas semiestruturadas permitiram um amplo volume de dados. O entrevistador foi orientado a seguir um roteiro de perguntas que buscavam estimular respostas quanto à avaliação do projeto pela perspectiva dos participantes, dando liberdade para os entrevistados se manifestarem livremente mesmo quando se desviassem do ponto central da pergunta. Desse modo, as entrevistas apresentaram grande diversidade de forma e 
conteúdo, variando, em tempo de gravação, entre 08 minutos e 23 segundos (a mais curta) até 43 minutos e 45 segundos (a mais longa).

A escolha dos entrevistados orientou-se pelos critérios de representatividade (que os participantes representassem diversos grupos de stakeholders do Projeto MARGov), de tipicidade (que as entrevistas contemplassem os participantes de maior envolvimento com o Projeto em seu grupo de stakeholders) e de acessibilidade (que o participante se dispusesse a avaliar o Projeto em entrevista).

Os respondentes se vinculam a seis grupos de stakeholders, quais sejam: 1) pescadores - 2 entrevistados; 2) membros de associação de turismo e lazer - 3 entrevistados, 3) pesquisadores / cientistas - 4 entrevistados; 4) trabalhadores na gestão do Parque enquanto servidores da administração pública - 3 entrevistados; 5) representantes do governo local - 3 entrevistados; e 6) servidores de instituições públicas nacionais - 2 entrevistados.

Não se distinguiu um grupo de stakeholders específicos para moradores locais, pois observou-se sobreposição desse atributo com outras características dos stakeholders. Dentre os 17 entrevistados, 8 deles (vinculados a cinco dos seis grupos entrevistados) residiam na região de Sesimbra e realçaram esse fato nas entrevistas. Apenas o grupo de gestores do Parque não conta nenhum residente em Sesimbra. Essa sobreposição de caraterística dos entrevistados (local de residência e grupo de vinculação) revela a concomitância de papeis sociais dos entrevistados, que condiz com a multiplicidade de perspectivas e coexistência de interesses nas questões relacionadas ao Parque.

\subsection{Tratamento dos dados}

O tratamento dos dados foi realizado com o auxílio do software webQDA de análise qualitativa (Sousa, Costa, \& Moreira, 2019). Os textos transcritos das entrevistas foram importados para a Plataforma webQDA em formato Word, como fontes reunidas em uma pasta específica. A partir da plataforma webQDA foi possível criar códigos a serem aplicados a excertos do texto para destacar as passagens relativas às categorias consideradas na análise.

Bardin (2016, p. 147) explica que, "a partir do momento em que a análise de conteúdo decide codificar seu material, deve produzir um sistema de categorias". O sistema de 
categorias deve refletir os objetivos da pesquisa. Assim, orientado pelo objetivo de analisar a avaliação dos participantes quanto aos processos e resultados do Projeto MARGov, distinguiram-se categorias que ajudaram a revelar o conteúdo das avaliações representadas nos textos das entrevistas. A unidade de codificação foi o enunciado, ou seja, unidade de significação que contemple o que é dito sobre o objeto de avaliação, incluindo as observações quanto o contexto do que está sob avaliação pelo entrevistado. Para a codificação dos dados, foram aplicadas três dimensões de análise.

A primeira dimensão é composta por categorias temáticas que permitiram identificar os principais temas abordados pelos entrevistados. Por meio da técnica de categorização por acervo (Bardin, 2016, p. 148), pela qual as categorias emergem ao longo da classificação analógica e progressiva dos textos, foram identificados 20 temas que configuram objeto de avaliação no texto das entrevistas. A codificação dos textos nessas categorias temáticas foi feita na plataforma webQDA pelo uso de códigos-árvore para permitir o desdobramento das categorias temáticas em subcategorias, quando necessário, gerando, assim, uma estrutura hierárquica entre os códigos. Vale ressaltar que, para essa primeira dimensão, as categorias não foram mutuamente excludentes, permitindo a multicodificação (codificação de uma mesma passagem do texto em duas ou mais categorias da mesma dimensão).

A segunda dimensão de análise distinguiu o julgamento; a apreciação e/ou o sentimento do entrevistado (positivo ou negativo) quanto aos temas relativos ao Projeto MARGov.

A terceira dimensão buscou identificar se os elementos avaliados pelos entrevistados constituíam em aspectos internos ao Projeto MARGov ou aspectos de contexto do Projeto que poderiam influenciar seu desenvolvimento (processos) e seus resultados. Juntas, a segunda e a terceira dimensões de análise compuseram uma grade de categorias avaliativas inspirada nos componentes da Análise SWOT.

Nesse caso, optou-se por utilizar códigos livres, pois essas categorias não presumem subcategorias.

Gürel e Tat (2017, p. 995) descrevem a Análise SWOT como uma técnica de "análise interacional que favorece macro avaliações" de organizações e/ou projetos ${ }^{3}$. Os autores ${ }^{3}$ Tradução livre e adaptação do original: "SWOT Analysis is an interactional analysis technique that makes macro
evaluations Possible". 
explicam que a Análise SWOT é uma ferramenta de diagnóstico situacional capaz de subsidiar a tomada de decisões estratégicas. Composta por uma matriz de tipo $2 \times 2$, a ferramenta da Análise SWOT - ou Matriz SWOT - distingue quatro quadrantes que avaliam aspectos positivos $\mathrm{X}$ negativos associados ao ambiente interno $\mathrm{X}$ externo do projeto.

Os aspectos internos podem ser trabalhados para o aprimoramento do projeto. Já os aspectos externos, normalmente associados a elementos de contexto, fogem ao controle da gestão do projeto, mas deve ser monitorado dado seu potencial de gerar impactos (positivos e negativos) sobre o projeto. As categorias avaliativas inspiradas na SWOT orientaram as análises com vistas a extrair dessas avaliações elementos úteis para orientar os futuros aprimoramentos e/ou replicação do Projeto.

\section{ANÁLISE DAS AVALIAÇÕES DO PROJETO MARGOV POR SEUS PARTICIPANTES}

Tão logo aplicadas as codificações nos textos que compuseram o corpus de análise, a partir de sucessivas leituras interpretativas, procedeu-se a análise da sobreposição das codificações nas categorias temáticas e das codificações nas categorias avaliativas, para se conhecer os principais temas contemplados nas avaliações pelos entrevistados.

Para tanto, foi gerada uma matriz pela ferramenta "questionamento -> matrizes" na plataforma webQDA.

Essa matriz cruzou as 20 categorias temáticas e suas subcategorias (nas linhas da matriz) e as 4 categorias inspiradas na SWOT (nas colunas da matriz) e revelou o número de fontes em que ocorreu a sobreposição de codificação dessas duas dimensões de análise.

Essa matriz foi, então, exportada para o MSOffice Excel onde se computou a ocorrência dos temas nas categorias avaliativas (positivo/negativo; externo/externo).

A Figura 1 sintetiza os resultados dessa análise, considerando o número de entrevistas em que se identificou a sobreposição de cada tema em sua relação como o respectivo quadrante da Matriz SWOT. 


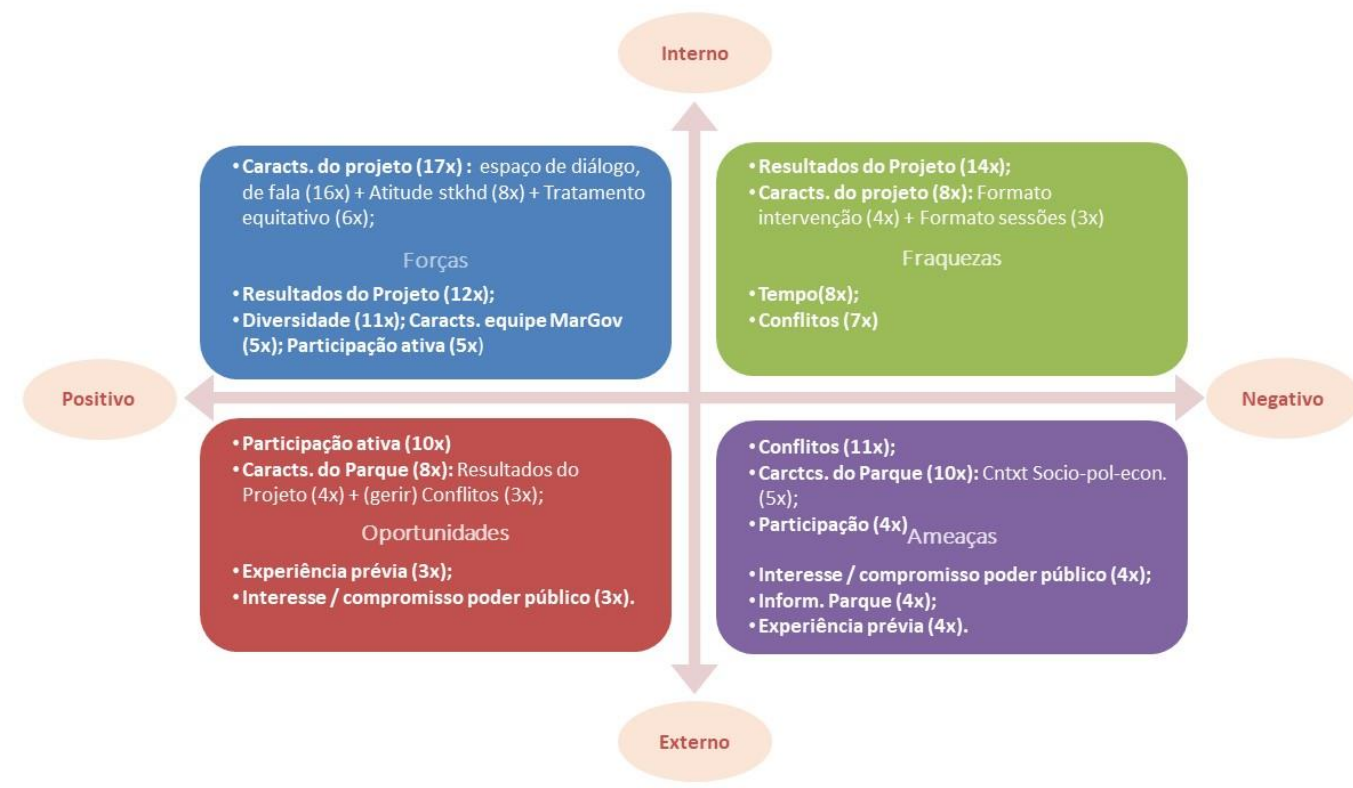

Figura 1. Distribuição dos temas avaliados pelos entrevistados segundo os quadrantes da Matriz SWOT

Fonte: Elaboração própria.

Observa-se que os entrevistados foram unânimes em reconhecer as características do Projeto MARGov como um aspecto positivo a ser realçado em sua avaliação. Dentre as características avaliadas positivamente pelos entrevistados, vale destacar que 16 respondentes apreciaram o Projeto por ser um espaço de diálogo e de fala. A diversidade dos participantes e a capacidade de gerar participação ativa também foram mencionadas como forças do projeto, bem como as características da própria equipe de facilitação. Ainda realçando elementos internos percebidos como positivos, tem-se que 12 respondentes consideram o Projeto enriquecedor, por oportunizar a informação e o aprendizado.

Essa apreciação positiva dos resultados do projeto parece contrastar com o primeiro item do quadrante "Fraquezas na Figura 1 em que (as lacunas de) os resultados do Projeto estão representados como sua principal fraqueza na perspectiva de 14 entrevistados. Esse contraste não se configura paradoxal pois, na avaliação dos respondentes, os resultados do Projeto foram avaliados em cinco subcategorias da categoria "Resultado". Dentre essas subcategorias, a "lacuna de resultados pragmáticos do Projeto" e a de "descontinuidade e retrocessos nos resultados obtidos" foram avaliados como aspetos internos negativos. A escassez de tempo nas discussões, as dificuldades de encaminhamento das atividades e das discussões devido a conflitos entre os stakeholders, e características do Projeto, como o 
formato das sessões (horário, local e frequência das reuniões e seu formato de desenvolvimento) também foram apontados como fraquezas do Projeto pelos participantes.

O principal elemento que caracteriza as oportunidades identificadas pelos entrevistados reside na motivação em participar da sociedade e, mais especificamente, dos stakeholders do Projeto. Porém, os conflitos foram apontados pelos respondentes como as principais ameaças ao Projeto MARGov. Nessa categoria ("Conflitos") computaram-se das avaliações negativas de conflitos entre os grupos de stakeholders - apontados como conflitos históricos no contexto do PMPLS, e mesmo os conflitos dentro do próprio grupo; bem como o conflito de interesses no que concerne à lida com o Parque que resultam, até mesmo, em conflitos com a lei quando se materializam em práticas irregulares e contravenções na área do Parque

$\mathrm{Na}$ sequência da análise da matriz do cruzamento das categorias temáticas com as categorias avaliativas, procedeu-se a análise de cobertura de codificação. Essa etapa analítica permitiu identificar a preponderâncias das avaliações dos textos dos respondentes segundo as categorias inspiradas na SWOT. As taxas de cobertura da fonte pela categoria (em valores percentuais, de modo a equalizar a variação de extensão das entrevistas) é gerada automaticamente pelo webQDA e podem ser visualizadas no canto direito da tela ao se consultar cada uma das categorias, como ilustra a Figura $2^{4}$.

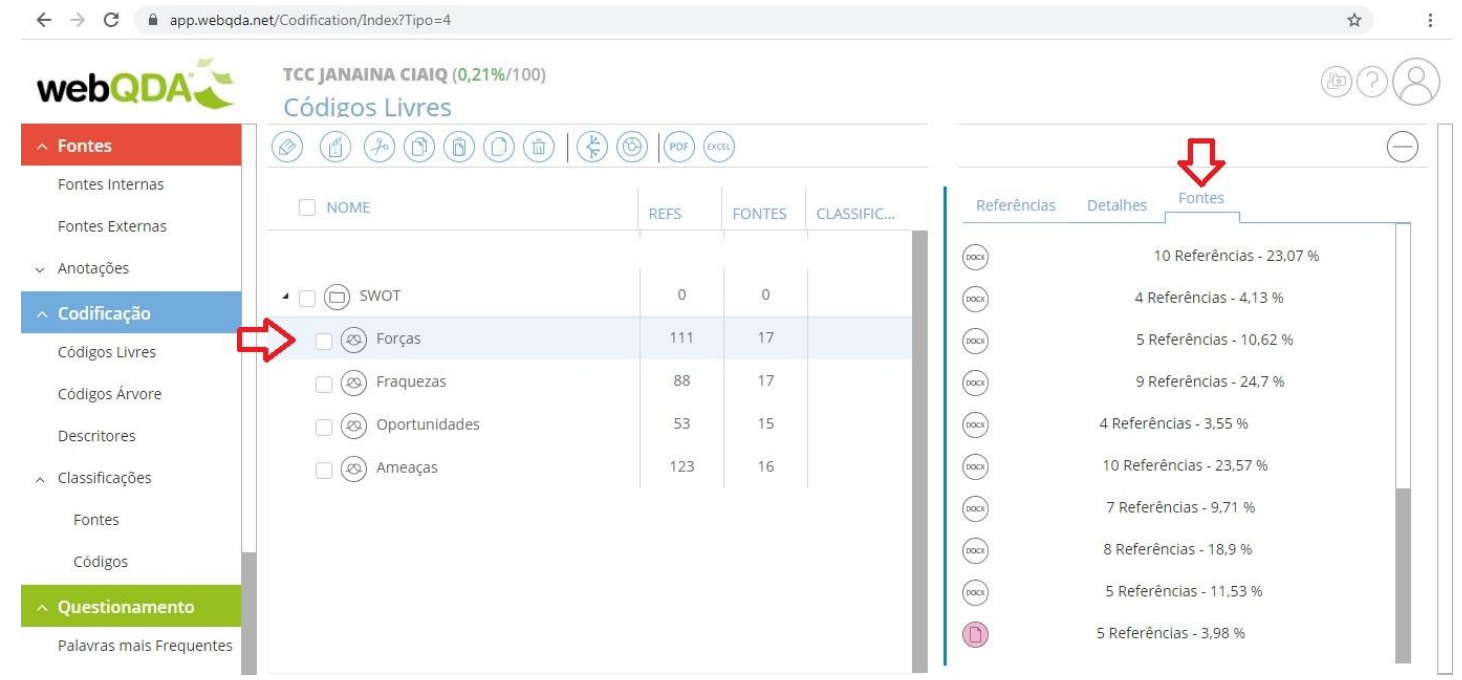

Figura 2. Indicadores de percentual de cobertura de codificação em cada fonte na plataforma webQDA. Fonte: Elaboração própria.

\footnotetext{
${ }^{4}$ A ilustração da tela foi modificada para realce dos elementos e supressão de informações de identificação pessoal do entrevistado
} 
Tais informações foram copiadas para um arquivo MS Office Excel para análise descritiva e geração dos gráficos que subsidiam as discussões que se seguem. A Figura 3 mostra o comparativo entre a cobertura de codificação dos textos das entrevistas nas categorias avaliativas.
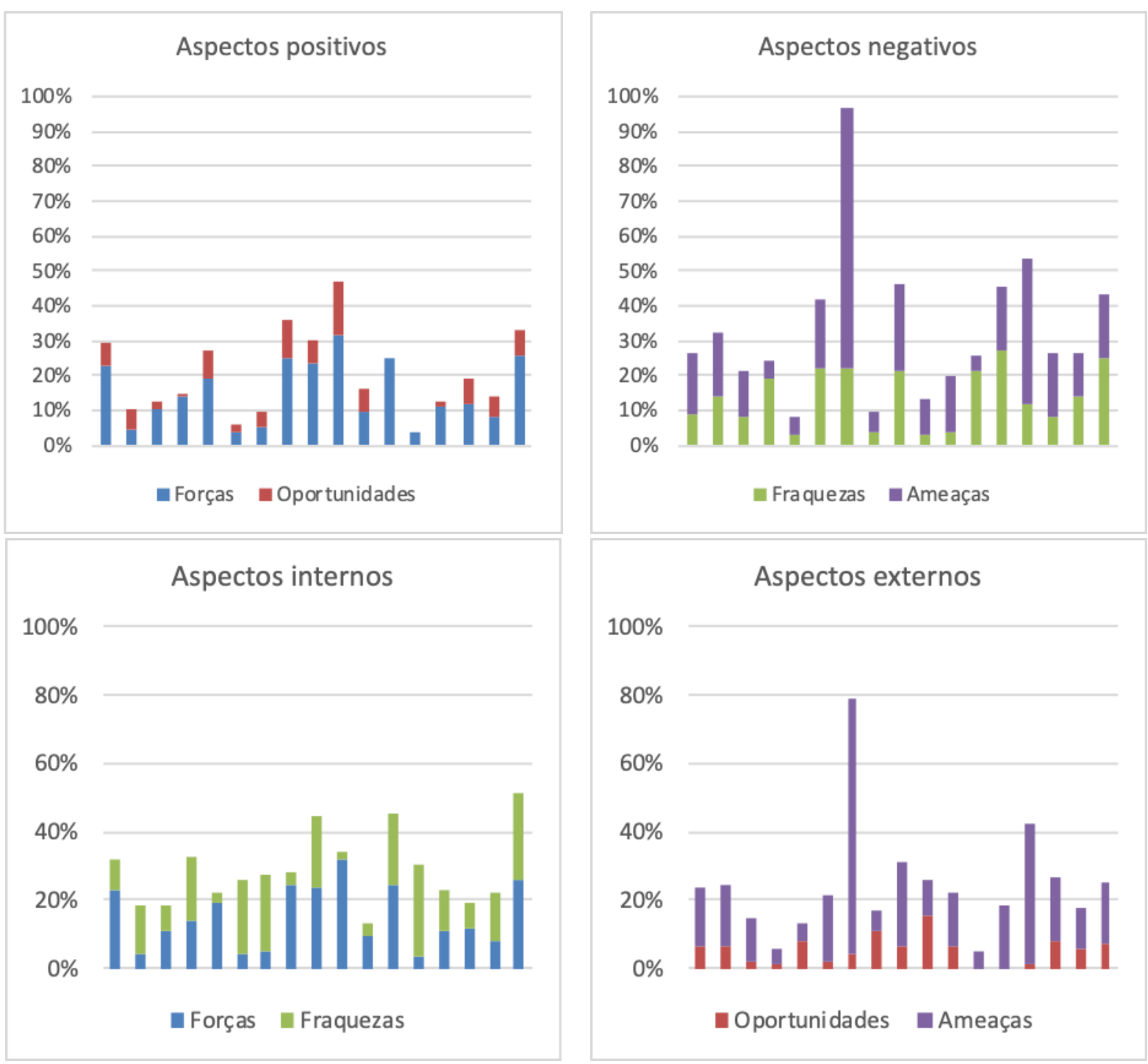

Figura 3. Comparativo das avaliações segundo categorias SWOT (por cobertura de codificação por entrevista). Fonte: Elaboração própria.

Pela prevalência das cores nos gráficos da Figura 3, é notável que, naquilo que concerne aos aspectos positivos mencionados pelos entrevistados, os aspectos internos (Forças) se sobressaem em relação aos aspectos externos (Oportunidades). Isso permite inferir que o Projeto MARGov, por suas características, é avaliado positivamente pelos entrevistados. Tal percepção é reiterada pela prevalência da cor azul (Forças) no gráfico relativo aos aspectos internos. Diferentemente, no que concerne aos aspectos externos, sobressai a avaliação 
negativa, representada pela cor violeta, revelando que, na percepção dos entrevistados, aspectos ambientais tiveram maior impacto negativo sobre os processos e os resultados do Projeto MARGov.

No cômputo geral, as avaliações negativas prevalecem nas entrevistas. O momento de escuta oportunizado pelas entrevistas foi uma oportunidade para os entrevistados expressarem suas frustrações, insatisfações e discordâncias nas questões relativas ao Projeto.

A prevalência de avaliações negativas nas entrevistas pode ser observada na Figura 4 que apresenta, por entrevista, a diferença (subtração) entre os percentuais da área de cobertura das categorias que denotam avaliação positiva (Forças + Oportunidade) e aqueles das categorias que denotam avaliação negativa (Fraquezas + Ameaças).

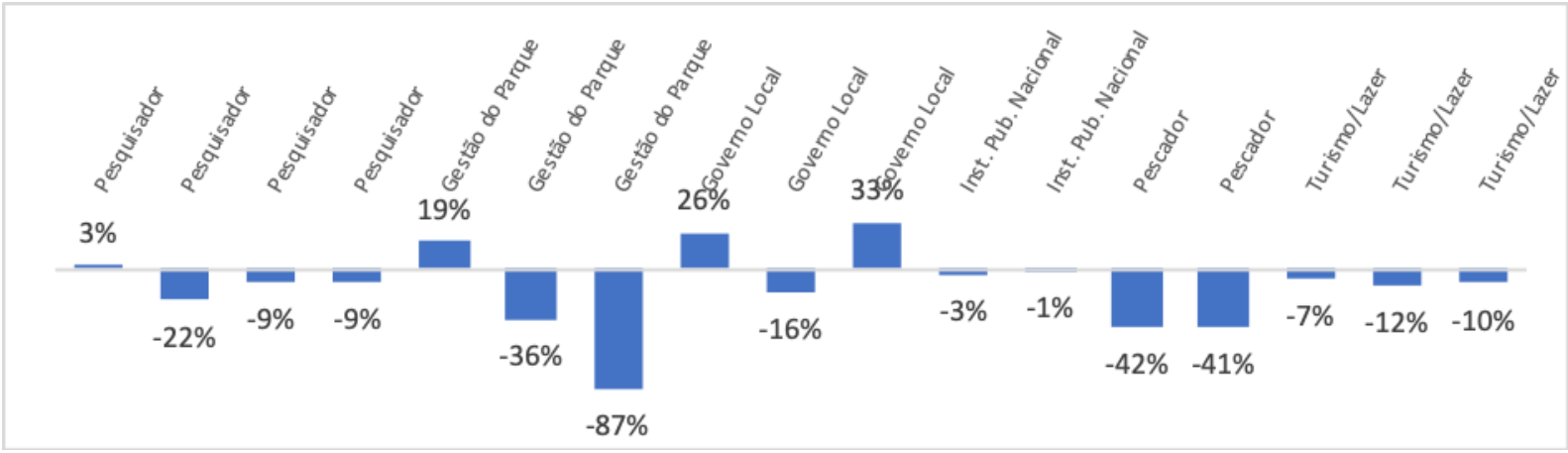

Figura 4. Diferença da cobertura de codificação entre categorias de conotação positiva e de conotação negativa (cômputo geral) nos textos dos entrevistados (por entrevista).

Fonte: Elaboração própria.

Porém, considerando apenas os aspectos internos ao Projeto MARGov, as avaliações são majoritariamente positivas, como se vê na Figura 5.

Apenas três entrevistados representam, em seus textos, mais aspectos negativos (por área de cobertura de codificação) do que positivos.

Pela comparação entre números (percentuais) indicados na Figura 4 e aqueles presentes na Figura 5, é possível afirmar que os aspectos internos contribuem pouco para esses números, de modo que os aspectos externos ao Projeto MARGov - isto é, os elementos de contexto constituem o objeto prioritário das avaliações negativas. 


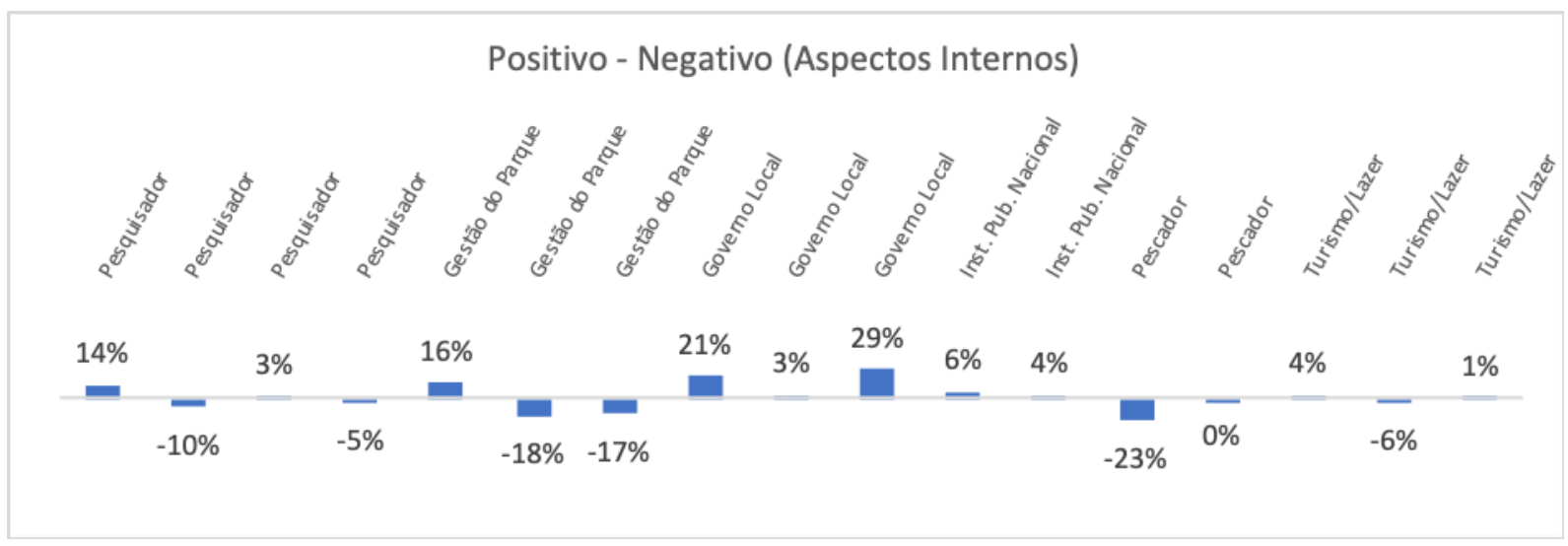

Figura 5. Diferença da cobertura de codificação de categorias de conotação positiva e categorias de conotação negativa (apenas aspectos internos ao Projeto MARGov) nos textos dos entrevistados (por entrevista).

Fonte: Elaboração própria.

Ainda que as avaliações negativas pareçam preponderantes nas entrevistas, na avaliação dos aspectos internos ao Projeto MARGov, a percepção positiva das "forças" do projeto sobressaiu-se na perspectiva dos entrevistados. Essa avaliação positiva denota reconhecimento dos participantes quanto ao valor do Projeto e contribui para a manutenção de seu engajamento. Os aspectos internos avaliados negativamente (fraquezas), apesar de não prevalecerem no cômputo geral, merecem atenção por representarem fragilidades que imputam riscos ao Projeto, podendo comprometer-lhe os processos e seus resultados.

Já ao que concerne aos aspectos externos avaliados pelos entrevistados, tem-se que eles pautam boa parte das avaliações negativas e dão a conhecer a medida do desafio a ser enfrentado pelos stakeholders nas questões relativas ao Projeto.

\section{CONSIDERAÇÕES FINAIS}

O desdobramento da primeira fase do Projeto MARGov em duas fases sucessivas de maturação e consolidação dos resultados é testemunha factual do sucesso do Projeto. Considerando que a manutenção do engajamento dos stakeholders é condicionado pelo reconhecimento do valor da iniciativa e de seus resultados, a análise apresentada neste trabalho revelou que o formato e metodologia do Projeto, a riqueza da diversidade dos atores envolvidos e os qualificadores da equipe facilitadora favoreceram a construção de uma comunidade de aprendizado mútuo, reconhecido pelos participantes como elemento crucial para esse sucesso. 
Não obstante, os participantes reconhecem fragilidades no Projeto capazes de alimentar um discurso desmotivador da participação, uma vez que uma parte significativa das medidas consensuadas têm levado o seu tempo em serem implementadas, embora uma parte substancial do que foi debatido nas sessões tenha vindo, gradualmente, a integrar a política de gestão do Parque. No entanto, pesam-se os paradoxos situacionais, por vezes históricos, como, no caso do MARGov, os antagonismos entre interesses de preservação ambiental, de maximização de benefícios na exploração de recursos naturais e de gestão de recursos de uso comum.

Os participantes reconhecem, também, que o contexto é favorável ao desenvolvimento de iniciativas colaborativas e que existe, na sociedade, um apelo pela participação social - o que é fato especialmente para as questões ligadas ao ambiente. Eis uma oportunidade importante para propiciar a construção de novos modelos de governança colaborativos. Mas também é inegável que conflitos (latentes ou manifestos) ameaçam os processos participativos e rouba-Ihes eficiência.

Para se avançar no aprimoramento do Projeto, é fundamental que as forças se compatibilizem com os aspectos externos, e que esses aspectos externos não venham incrementar as fraquezas do Projeto. A percepção, pelos stakeholders, dessa compatibilidade contribui para a percepção do valor da iniciativa, favorecendo a manutenção de seu engajamento, o que, por sua vez, é requisito para resultados profícuos em um processo colaborativo. A análise discursiva da avaliatividade nos textos dos entrevistados pode complementar os resultados da análise realizada no escopo deste trabalho.

\section{REFERÊNCIAS}

Bardin, L. (2016). Análise de conteúdo. São Paulo: Edições 70.

Carneiro, G. (Oct - Dec de 2011). The Luiz Saldanha Marine Park: an overview of conflicting perceptions. Conservation and Society, 9(4), 325-333. doi:10.4103/0972-4923.92149

Gonçalves, E. J., Serrão, E. A., Erzini, K., Henriques, M., Cunha, A. H., Horta e Costa, B., . . Henriques, V. (2015). Implementação do Parque Marinho Professor Luiz Saldanha (Parque Natural da Arrábida): Ponto de situação realizado no âmbito do Projeto de conservação e gestão BIOMARES. doi:10.13140/2.1.4302.1601

Gürel, E., \& Tat, M. (August de 2017). SWOT Analysis: a theoretical review. Journal of International Social Research, 10(51), pp. 994-1006. doi:10.17719/jisr.2017.1832

Ministério do Ambiente. (1998). Decreto Regulamentar n.ํ23/98. Diário da República n.ำ237/1998, Série I-B de 1998-10-14. Lisboa, Portugal: Diário da República Eletrônico. Acesso em 25 de fev de 2020, disponível em https://dre.pt/application/conteudo/234913 
Sousa, F. N., Costa, A., \& Moreira, A. (2019). webQDA [software]. Aveiro: Microio/Ludomedia.

Stratoudakis, Y., Farrall, H., \& Vasconcelos, L. (2018). Collaborative lessons towards marine sustainability: a long-term collective engagement. Sustainability Science, pp. 1-14. doi:http://dx.doi.org/10.1007/s11625018-0610-1

Stratoudakis, Y., Fernández, F., Henriques, M., Martins, J., \& Martins, R. (2015). Situação ecológica, socioeconómica e de governança após a implementação do primeiro plano de ordenamento no Parque Marinho Professor Luiz Saldanha (Arrábida, Portugal): II - percepção dos utentes. Revista de Gestão Costeira Integrada / Journal of Integrated Coastal Zone Management, 2, pp. 167 - 178. doi:10.5894/rgci578

Thomson, A., \& James, P. (2006). Collaboration Processes: Inside the Black Box. Public Administration Review, 66, pp. 20-32. doi:10.1111/j.1540-6210.2006.00663.x

United Nations. (1992). Agenda 21. United Nations Conference on Environment and Development. Rio de Janeiro: United Nations.

United Nations. (1992). Rio Declaration on Environment and Development. United Nations Conference on Environment and Development. Rio de Janeiro (Brasil): United Nations.

United Nations General Assembly. (1972). Report of United Nations Conference on the Human Environment. United Nations Conference on the Human Environment. Stockholm (Sweden): United Nations.

United Nations General Assembly. (2012). The Future we Want: outcome of the United Nations Conference on Sustainable Development. United Nations Conference on Sustainable Development - Rio+20). Rio de Janeiro: United Nations.

Vasconcelos, L., Coelho, M. R., Pereira, M. R., Sá, R., \& Costa, M. (2011). PROJECTO MARGov: Governância Colaborativa de Áreas Marinhas Protegidas O Diálogo Eco-Social na Capacitação de Agentes de Mudança para a Sustentabilidade dos Oceanos - Relatório Final Parte A. Relatório Técnico de Pesquisa, IMAR Instituto do Mar / DCEA / FCT / UNL, Lisboa. Acesso em 21 de ago de 2019, disponível em https://www.researchgate.net/publication/266672413_PROJECTO_MARGov_Governancia_Colaborativa_d e_Areas_Marinhas_Protegidas_O_Dialogo_Eco-

Social_na_Capacitacao_de_Agentes_de_Mudanca_para_a_Sustentabilidade_dos_Oceanos_O_caso_de_ estudo_do_Parque_Marinho_P

WTeamUp. (2019). MARGov - Governância Colaborativa de Áreas Marinhas Protegidas. WTeam Participação Empowerment: https://www.wteamup.com/margov. 